\title{
Photoabsorption spectra in the continuum of molecules and atomic clusters
}

\author{
Takashi Nakatsukasa* \\ RI Beam Science Laboratory, RIKEN, 2-1 Hirosawa, Wako 351-0198, Japan \\ Kazuhiro Yabana ${ }^{\dagger}$ \\ Institute of Physics, University of Tsukuba, Tennodai 1-1-1, Tsukuba 305-8571, Japan.
}

\begin{abstract}
We present linear response theories in the continuum capable of describing photoionization spectra and dynamic polarizabilities of finite systems with no spatial symmetry. Our formulations are based on the time-dependent local density approximation with uniform grid representation in the three-dimensional Cartesian coordinate. Effects of the continuum are taken into account either with a Green's function method or with a complex absorbing potential in a real-time method. The two methods are applied to a negatively charged cluster in the spherical jellium model and to some small molecules (silane, acetylene and ethylene).
\end{abstract}

PACS number(s): 31.15.Ew, 31.70.Hq, 33.80.Eh

\section{INTRODUCTION}

Oscillator strength distribution characterizes the optical response of atoms and molecules. Advances in measurements with synchrotron radiation and high resolution electron energy loss spectroscopy have enabled us to obtain oscillator strength distribution of a whole spectral region originated from valence electrons [1]. The photon energy dependences of molecular photoelectron spectra have also been measured. They provide useful information about the dynamics of the photoionization processes.

Theoretical analysis of photoabsorption spectra above the first ionization threshold requires continuum electronic wave function in a non-spherical multi-center potential. Several methods have been developed for this purpose [2], including the continuum multiple scattering method [3], the Schwinger variational method [4.5], finite-volume variational method [6], the linear algebraic method [7], and the $K$-matrix method [8]. The Stieltjes moment method does not directly utilize the scattering state but extract continuum spectrum from the spectral moments which are calculated with a square-integrable basis set [9]. The method has been extensively applied to large systems 10,11. The complex basis method also gives continuum spectra by the calculation with a squareintegrable basis set [12].

The purpose of the present article is to present alternative theoretical methods for the continuum spectra of molecules. Our methods rely upon the linear response theory in the time-dependent local-density approximation (TDLDA) and employ a uniform grid representation in a three-dimensional Cartesian coordinate.

The TDLDA (or alternatively called the timedependent density-functional theory) is an extension of the static density-functional theory to describe electronic dynamics under a time-dependent external field 13]. In the practical applications, an adiabatic approximation is usually assumed: the same exchange-correlation potential as that in the static case is used for the timedependent problem. The correlation effect is included through the dynamical screening which is represented by an induced local potential. In the TDLDA, the continuum boundary condition was treated with the radial Green's function for spherical systems such as atoms 114 and clusters in the spherical jellium model [15, 16]. The importance of the dynamical screening effect on the continuum response has been stressed. The method has been extended for molecules with a single-center expansion technique 17. However, the application was limited to small, axially symmetric molecules.

Since the Hamiltonian in the Kohn-Sham theory is almost diagonal in coordinate representation, a grid representation in the coordinate space provides an economical description. A uniform grid representation in the three-dimensional Cartesian coordinate which has been developed by Chelikowsky et.al [18] provides a convenient basis for our purpose. The problem is then how to incorporate the scattering boundary condition in the uniform grid representation.

Our first method is based on the real-time method which one of the present authors has recently developed to calculate dynamic polarizability of finite systems 19.20]. In the method, the time-dependent Kohn-Sham equation is solved explicitly in real-time as well as in realspace with a uniform grid. The dynamic polarizability of a whole spectral region is then obtained at once through the time-frequency Fourier transformation. An advantage of this method is that we do not need to handle

\footnotetext{
*Email: Ntakashi@riken.go.jp

${ }^{\dagger}$ Email: yabana@nucl.ph.tsukuba.ac.jp
} 
matrices of very large dimensionality, since only a single Slater determinant is evolved in time. A drawback is that the single-particle continuum states cannot be treated exactly because the electrons are confined in a limited size of box (model space). A possible way to avoid the difficulty would be employing a complex absorbing potential at the end of the box, which we shall discuss later.

Our second method is based on the modified Sternheimer method 21]. The method has been widely used in the linear response calculations [22,23]. It recasts the response problem into solving the static Schrödinger-type equation with a source term. The problem is then to solve the equation with an appropriate outgoing boundary condition. Our recipe here is to solve iteratively the equation taking into account the boundary condition employing a Green's function of a spherical potential, separating the self-consistent potential into long range spherical and short range non-spherical parts.

The paper is organized as follows. We present our methods in Sec. [II. The real-time method with an absorptive boundary potential and the Green's function method are explained. In Sec. III, applications of the methods will be demonstrated. First the spherical jellium cluster is considered to confirm reliability of our methods. We then show calculations of photoabsorption spectra of some small molecules and compare them with measurements. We give interpretation of the obtained spectra. Finally conclusion are drawn in Sec. IV.

\section{LINEAR RESPONSE IN THE CONTINUUM OF THREE-DIMENSIONAL REAL SPACE}

\section{A. Real-time method with an absorbing potential}

In this section we first recapitulate the real-time method in TDLDA 19,24. For systems with relatively large number of particles, the real-time method is one of the most efficient method to calculate the electronic excitations in molecules and atomic clusters.

The TDLDA equations for a spin-independent $N$ electron system are given in terms of the time-dependent Hamiltonian $h(t)$ which is a functional of the density $n(\mathbf{r}, t)$.

$$
\begin{aligned}
& i \frac{\partial}{\partial t} \phi_{i}(\mathbf{r}, t)=h(t) \phi_{i}(\mathbf{r}, t), \quad i=1, \cdots, N / 2 \\
& h(t)=-\frac{1}{2 m} \nabla^{2}+V_{\text {ion }} \\
& \quad+e^{2} \int d^{3} r^{\prime} \frac{n\left(\mathbf{r}^{\prime}, t\right)}{\left|\mathbf{r}-\mathbf{r}^{\prime}\right|}+\mu_{\mathrm{xc}}[n(\mathbf{r}, t)], \\
& n(\mathbf{r}, t)=2 \sum_{i=1}^{N / 2}\left|\phi_{i}(\mathbf{r}, t)\right|^{2} .
\end{aligned}
$$

We adopt $\hbar=1$ throughout the present paper. $V_{\text {ion }}$ is an electron-ion potential for which we employ a norm- conserving pseudopotential [25] with a separable approximation [26]. $\mu_{\mathrm{xc}}$ is an exchange-correlation potential.

We represent the electronic wave function on a uniform grid points inside a certain spatial area [18]. All potentials in Eq. (2.2) are diagonal in this representation except for the nonlocal part of $V_{\text {ion }}$. The second order differential operator in the kinetic energy is approximated employing a nine-point formula. For the time evolution, we use an algorithm developed in Ref. [27] which utilize a predictor-corrector method. The integration of Eq. (2.1) is approximately carried out with a fourth-order Taylor expansion.:

$$
\begin{aligned}
\phi_{i}(t+\Delta t) & =e^{-i h(t+\Delta t / 2) \Delta t} \phi_{i}(t) \\
& \approx \sum_{n=0}^{4} \frac{(-i h(t+\Delta t / 2) \Delta t)^{n}}{n !} \phi_{i}(t) .
\end{aligned}
$$

For stable iteration, the time step $\Delta t$ should be smaller than the inverse of the maximum eigenvalue of the Hamiltonian. The energy and the particle-number conservations are then well satisfied. This method was successful in nuclear physics to investigate the dynamics of nuclear reaction 27,28 and has been proved to be fruitful for electron systems as well [19,24].

We start with the static LDA problem solving the ground-state Kohn-Sham equations and determine the initial occupied orbitals $\phi_{i}(\mathbf{r})$ and density $n_{0}(\mathbf{r})$. The conjugate gradient method is used to solve the static Kohn-Sham equation. Then, an external field is turned on instantaneously at $t=0$. In this paper, we shall consider only the dipole response, adopting $V_{\text {ext }}=k_{0} r_{\nu} \delta(t)$ $(\nu=x, y, z)$ which produces an initial state for the time evolution as

$$
\phi_{i}\left(\mathbf{r}, 0_{+}\right)=e^{-i k_{0} r_{\nu}} \phi_{i}(\mathbf{r}), \quad \nu=x, y, z .
$$

We calculate time evolution with this initial condition without any external fields. The dynamical polarizabilities in the time representation is then obtained as

$$
\alpha_{\nu}(t)=-\frac{e^{2}}{k_{0}} \int d^{3} r r_{\nu} \delta n(\mathbf{r}, t), \quad \nu=x, y, z,
$$

where the transition density is simply given by the difference from that of the ground-state

$$
\delta n(\mathbf{r}, t)=n(\mathbf{r}, t)-n_{0}(\mathbf{r})
$$

Since the dynamical polarizability is usually defined in the energy representation, we take the Fourier transform of Eq. (2.6).

$$
\alpha_{\nu}(\omega)=\int_{0}^{T} d t \alpha_{\nu}(t) e^{i \omega t-\Gamma t / 2}, \quad \nu=x, y, z,
$$

where we introduce a smoothing parameter $\Gamma$. In order to obtain $\alpha(\omega)$ in energy resolution of $\Gamma$, we need to calculate the time evolution up to $T \geq 2 \pi / \Gamma$. In the next 
section, we discuss a gas-phase average of the $x, y$ and $z$ direction, as

$$
\alpha(\omega)=1 / 3 \sum_{\nu=x, y, z} \alpha_{\nu}(\omega)
$$

When the energy is in a region where $\omega+\epsilon_{i}>0$, a part of the electron wave functions $\phi_{i}(\mathbf{r}, t)$ can escape to the continuum. This outgoing part of wave function is eventually reflected at the edge of the box and produces a spurious discrete structure in the photoabsorption spectra. Therefore, we now introduce an imaginary absorbing potential $W(\mathbf{r})$ to suppress the reflection and mimic the continuum. Since the imaginary potential should be zero in a region where the ground-state density has a finite value, we should adopt a spherical box of large radius and the imaginary potential is switched on only in a outer shell of width $\Delta r$. Although the complex potential at the edge of the box slightly violates normalization and energy conservation, the box must be large enough to preserve the conservations if the time evolution is carried out using an initial state of Eq. (2.5) with $k_{0}=0$ (the ground state).

We adopt an absorptive potential of a linear dependence on coordinate, which has been discussed in the wave-packet method for molecular collisions [29,30]:

$$
W(r)= \begin{cases}0 & \text { for } 0<r<R \\ -i W_{0} \frac{r-R}{\Delta r} & \text { for } R<r<R+\Delta r\end{cases}
$$

The height $W_{0}$ and width $\Delta r$ must be carefully chosen to prevent the reflection. There has been an argument based on the WKB theory to elucidate a condition of no reflection [29, 30].

For simplicity, let us take a one-dimensional system. The absorbing potential is given by a form similar to Eq. (2.10): $W(x)=0$ for $x<0$ and $W(x)=-i W_{0} x / \Delta x$ for $x>0$. The WKB solution is

$$
\begin{aligned}
& \psi(x)= \exp (i k x)+R \exp (-i k x) \quad \text { for } x<0, \\
& \psi(x)=(1+R) E^{1 / 4}\left(E+i W_{0} \frac{x}{\Delta x}\right)^{-1 / 4} \\
& \quad \times \exp \left\{i \int_{0}^{x} d x k(x)\right\} \quad \text { for } x>0,
\end{aligned}
$$

where $k(x)=\left[2 m\left(E+i W_{0} x / \Delta x\right)\right]^{1 / 2}$ and $k=k(0)$. Conditions we require are $|R|^{2} \ll 1$ (no reflection) and $|\psi(\Delta x)| \ll 1$ (complete absorption). A continuity condition for a derivative at $x=0$ leads to

$$
R \approx \frac{W_{0}}{4 \sqrt{8 m} E^{3 / 2} \Delta x}
$$

assuming $W_{0} / E \ll 1$. The absolute value of wave function at $x=\Delta x$ is approximately given by

$$
|\psi(\Delta x)| \approx \exp \left\{-W_{0} \Delta x\left(\frac{m}{8 E}\right)^{1 / 2}\right\}
$$

If we demand $|R|^{2}<0.001$ and $|\psi(\Delta x)|^{2}<0.01$, we have

$$
20 \frac{E^{1 / 2}}{\Delta r \sqrt{8 m}}<\left|W_{0}\right|<\frac{1}{10} \Delta r \sqrt{8 m} E^{3 / 2},
$$

where $\Delta x$ has been replaced by $\Delta r$. This is the condition discussed in Ref. 30 which we shall test with numerical calculations. Strictly speaking, the conditions, $|R|^{2}<0.001$ and $|\psi(\Delta x)|^{2}<0.01$, lead to a factor 18.4 instead of 20 in the left hand side and 0.128 instead of $1 / 10$ in the right hand side. In actual real-time calculations, electrons with different energies are emitted simultaneously. Thus, $\Delta r$ and $W_{0}$ must be chosen properly according to the energy spectrum of photoabsorption above the ionization threshold.

\section{B. Green's function method with an outgoing boundary condition}

Exact treatment of the continuum is possible with the use of a Green's function. For spherical systems, the Green's function can easily be constructed by making a multipole expansion and discretizing the radial coordinate. The method was first applied to nuclear giant resonances [31, 32] and then applied to photoabsorption in atoms [33]14]. In this section we present a method to construct a Green's function in the uniform grid representation for a system without any spatial symmetry.

The linear response theory is formulated most conveniently using a density-density correlation function [34]. The general formalism with local-density approximation is given in the frequency representation 32, 14. The transition density, which corresponds to the Fourier transform of Eq. (2.7), can be expressed with the use of the independent-particle density-density correlation function $\chi_{0}$ :

$$
\delta n(\mathbf{r}, \omega)=\int d^{3} r^{\prime} \chi_{0}\left(\mathbf{r}, \mathbf{r}^{\prime} ; \omega\right) V_{\mathrm{scf}}\left(\mathbf{r}^{\prime}, \omega\right),
$$

where $V_{\text {scf }}$ is the self-consistent field which is the sum of the external field and the dynamical screening (dielectric) field:

$$
V_{\mathrm{scf}}(\mathbf{r}, \omega)=V_{\mathrm{ext}}(\mathbf{r}, \omega)+\left.\int d^{3} r^{\prime} \frac{\delta V[n(\mathbf{r})]}{\delta n\left(\mathbf{r}^{\prime}\right)}\right|_{n=n_{0}} \delta n\left(\mathbf{r}^{\prime} ; \omega\right),
$$

where $V[n(\mathbf{r})]$ is a single-particle potential of the timeindependent version of Eq. (2.2), defined by $h=$ $-\nabla^{2} / 2 m+V$. Although a part of $V$ is non-local, the screening field arises from density-dependent parts of $V$, the direct Coulomb and the exchange-correlation potentials, which are local. The calculation neglecting the second term of Eq. (2.17) will be called an "independentparticle approximation" (IPA) in the next section.

The $\chi_{0}$ has a form 


$$
\begin{aligned}
\chi_{0}\left(\mathbf{r}, \mathbf{r}^{\prime} ; \omega\right)= & 2 \sum_{i}^{\text {occ unocc }} \sum_{m}\left\{\phi_{i}(\mathbf{r}) \frac{\phi_{m}^{*}(\mathbf{r}) \phi_{m}\left(\mathbf{r}^{\prime}\right)}{\epsilon_{i}-\epsilon_{m}-\omega-i \eta} \phi_{i}^{*}\left(\mathbf{r}^{\prime}\right)\right. \\
& \left.+\phi_{i}^{*}(\mathbf{r}) \frac{\phi_{m}(\mathbf{r}) \phi_{m}^{*}\left(\mathbf{r}^{\prime}\right)}{\epsilon_{i}-\epsilon_{m}+\omega+i \eta} \phi_{i}\left(\mathbf{r}^{\prime}\right)\right\}, \quad
\end{aligned}
$$

where $\eta$ is an infinitesimal positive parameter. The factor two in the right hand side of Eq. (2.18) is the spin degeneracy of each single-particle state. Here the summation with respect to unoccupied states $m$ contains both discrete and continuum states. Instead of taking the explicit summation, we may use the single-particle Green's function defined by

$$
\begin{aligned}
G^{( \pm)}\left(\mathbf{r}, \mathbf{r}^{\prime} ; E\right) & =\left\langle\mathbf{r}\left|\left(E-h\left[n_{0}\right] \pm i \eta\right)^{-1}\right| \mathbf{r}^{\prime}\right\rangle \\
& =\sum_{k}^{\text {all }} \frac{\phi_{k}(\mathbf{r}) \phi_{k}^{*}\left(\mathbf{r}^{\prime}\right)}{E-\epsilon_{k} \pm i \eta} .
\end{aligned}
$$

The sign determines a boundary condition of the Green's function; the $(+)$ for a outgoing wave and the $(-)$ for an incoming wave. In fact, the summation with respect to $m$ in Eq. (2.18) can be extended to all states because the summation over the occupied states for the first term will be canceled by a contribution from the second term. Therefore, using Eq. (2.19), we can rewrite (2.18) as

$$
\begin{aligned}
\chi_{0}\left(\mathbf{r}, \mathbf{r}^{\prime} ; \omega\right)= & 2 \sum_{i}^{\text {occ }} \phi_{i}(\mathbf{r})\left\{\left(G^{(+)}\left(\mathbf{r}, \mathbf{r}^{\prime} ;\left(\epsilon_{i}-\omega\right)^{*}\right)\right)^{*}\right. \\
& \left.+G^{(+)}\left(\mathbf{r}, \mathbf{r}^{\prime} ; \epsilon_{i}+\omega\right)\right\} \phi_{i}\left(\mathbf{r}^{\prime}\right)
\end{aligned}
$$

where we assume that the occupied states have real wave functions.

To calculate the dipole photoresponse of a system, one should take $V_{\text {ext }}=r_{\nu}, \nu=x, y, z$. Then, the dynamic polarizability is given by

$$
\alpha_{\nu}(\omega)=-e^{2} \int d^{3} r_{\nu} \delta n(\mathbf{r}, \omega), \quad \nu=x, y, z .
$$

Since the number of spatial grid points is large, it is not convenient and even impossible to construct explicitly the response function $\chi_{0}\left(\mathbf{r}, \mathbf{r}^{\prime} ; \omega\right)$ and to perform spatial integration by summing up over grid points in solving the self-consistent equations, Eqs. (2.16) and (2.17). We can avoid the difficulty by converting the integral into the equivalent differential equation. We introduce a function

$\psi_{i}\left(\mathbf{r} ; E, V_{\mathrm{scf}}\right) \equiv \int d^{3} r^{\prime} G^{(+)}\left(\mathbf{r}, \mathbf{r}^{\prime} ; E\right) V_{\mathrm{scf}}\left(\mathbf{r}^{\prime}\right) \phi_{i}\left(\mathbf{r}^{\prime}\right)$.

The transition density is then expressed as

$$
\begin{aligned}
\delta n(\mathbf{r}, \omega)=2 \sum_{i}^{\text {occ }} \phi_{i}(\mathbf{r})\{ & \left(\psi_{i}\left(\mathbf{r} ;\left(\epsilon_{i}-\omega\right)^{*}, V_{\mathrm{scf}}^{*}\right)\right)^{*} \\
& \left.+\psi_{i}\left(\mathbf{r} ; \epsilon_{i}+\omega, V_{\mathrm{scf}}\right)\right\} .
\end{aligned}
$$

When the energy $\omega$ is far below the first ionization threshold, all the outgoing channels are closed and Eq. (2.22) is equivalent to the Schrödinger equation of energy $E=$ $\epsilon_{i} \pm \omega$ with a source term,

$$
\left(E-h\left[n_{0}\right]\right)\left|\psi_{i}\right\rangle=V_{\mathrm{scf}}\left|\phi_{i}\right\rangle,
$$

assuming that the function $\left\langle\mathbf{r} \mid \psi_{i}\right\rangle$ outside a box area vanishes. The integral in Eq. (2.22) is thus converted into a linear algebraic equation (2.24). This procedure is known as the modified Sternheimer method [21]. However, since this cannot describe a correct asymptotic behavior of the wave function, it is not applicable when the energy $E$ is close to zero. Furthermore, when $E>0$, the method is incapable to produce the continuum spectra.

Thus, the remaining task is to calculate $\psi_{i}\left(\mathbf{r} ; E, V_{\mathrm{scf}}\right)$ defined by Eq. (2.22) under an appropriate outgoing boundary condition. For this purpose, we employ an integral equation for the Green's function. We start with a single-particle problem with a spherical potential $V_{0}(\mathbf{r})$. For instance, the Green's function for free particles $\left(V_{0}(\mathbf{r})=0\right)$ has an analytic expression,

$$
G_{0}^{(+)}\left(\mathbf{r}, \mathbf{r}^{\prime} ; E\right)=-\frac{m}{2 \pi} \frac{e^{+i k\left|\mathbf{r}-\mathbf{r}^{\prime}\right|}}{\left|\mathbf{r}-\mathbf{r}^{\prime}\right|}
$$

where $k=\sqrt{2 m E}(E>0)$. For a negative energy $E<0$, $\exp \left(+i k\left|\mathbf{r}-\mathbf{r}^{\prime}\right|\right)$ in Eq. (2.25) is replaced by $\exp \left(-\kappa\left|\mathbf{r}-\mathbf{r}^{\prime}\right|\right)$ with $\kappa=\sqrt{-2 m E}$. The single-particle Green's function for a non-spherical (even non-local) potential $V\left(\mathbf{r}, \mathbf{r}^{\prime}\right)$ then satisfies the following integral equation

$$
\begin{aligned}
& G^{(+)}\left(\mathbf{r}, \mathbf{r}^{\prime} ; E\right)=G_{0}^{(+)}\left(\mathbf{r}, \mathbf{r}^{\prime} ; E\right)+\int d^{3} r^{\prime \prime} d^{3} r^{\prime \prime \prime} \\
& G_{0}^{(+)}\left(\mathbf{r}, \mathbf{r}^{\prime \prime} ; E\right) \tilde{V}\left(\mathbf{r}^{\prime \prime}, \mathbf{r}^{\prime \prime \prime}\right) G^{(+)}\left(\mathbf{r}^{\prime \prime \prime}, \mathbf{r}^{\prime} ; E\right),
\end{aligned}
$$

where $\tilde{V}\left(\mathbf{r}, \mathbf{r}^{\prime}\right)=V\left(\mathbf{r}, \mathbf{r}^{\prime}\right)-V_{0}(\mathbf{r}) \delta^{3}\left(\mathbf{r}-\mathbf{r}^{\prime}\right)$. The boundary condition of $G_{0}^{(+)}$determines an asymptotic behavior of $G^{(+)}$. Substituting this into Eq. (2.22), we obtain a multi-linear equations for $\psi_{i}\left(\mathbf{r} ; E, V_{\mathrm{scf}}\right)$,

$$
\begin{aligned}
\psi_{i}(\mathbf{r} ; E & \left., V_{\mathrm{scf}}\right) \\
& -\int d^{3} r^{\prime} d^{3} r^{\prime \prime} G_{0}^{(+)}\left(\mathbf{r}, \mathbf{r}^{\prime} ; E\right) \tilde{V}\left(\mathbf{r}^{\prime}, \mathbf{r}^{\prime \prime}\right) \psi_{i}\left(\mathbf{r}^{\prime \prime} ; E, V_{\mathrm{scf}}\right) \\
& =\int d^{3} r^{\prime} G_{0}^{(+)}\left(\mathbf{r}, \mathbf{r}^{\prime} ; E\right) V_{\mathrm{scf}}\left(\mathbf{r}^{\prime}\right) \phi_{i}\left(\mathbf{r}^{\prime}\right) .
\end{aligned}
$$

In solving this equation, we need to evaluate the following integral

$$
\Psi(\mathbf{r} ; E)=\int d^{3} r^{\prime} G_{0}^{(+)}\left(\mathbf{r}, \mathbf{r}^{\prime} ; E\right) f\left(\mathbf{r}^{\prime}\right),
$$

where $f(\mathbf{r})$ is either $\int d^{3} r^{\prime} \tilde{V}\left(\mathbf{r}, \mathbf{r}^{\prime}\right) \psi_{i}\left(\mathbf{r}^{\prime} ; E, V_{\mathrm{scf}}\right)$ or $V_{\text {scf }}(\mathbf{r}) \phi_{i}(\mathbf{r})$. We note that both of them are zero outside the box. We calculate $\Psi(\mathbf{r} ; E)$ again by recasting Eq. (2.28) into equivalent differential equation,

$$
\left\{E-\left(-\frac{1}{2 m} \nabla^{2}+V_{0}(\mathbf{r})\right)\right\} \Psi(\mathbf{r} ; E)=f(\mathbf{r}) .
$$


In the discretization, this is a linear algebraic equation for grid points inside the box area. However, the solution outside the box area is needed to apply the Laplacian operator and should be prepared by other method. We prepare it by a multipole expansion method. Noting that $V_{0}(\mathbf{r})$ is a central potential, $G_{0}^{(+)}$can be expressed in terms of the regular and irregular solutions of radial Schrödinger equation in the usual way.

$$
\begin{gathered}
\left.\Psi(\mathbf{r} ; E)\right|_{r \geq R}=\sum_{l m}^{l_{\max }} \frac{w_{l}^{(+)}(r ; E)}{r} Y_{l m}(\hat{r}) \Phi_{l m}(E), \\
\Phi_{l m}(E) \equiv 2 m \int_{r<R} d^{3} r^{\prime} \frac{u_{l}\left(r^{\prime} ; E\right)}{r^{\prime}} Y_{l m}\left(\hat{r}^{\prime}\right) f\left(\mathbf{r}^{\prime}\right),
\end{gathered}
$$

where $u_{l}(r ; E)$ and $w_{l}^{(+)}(r ; E)$ are solutions of the radial differential equation being normalized as the Wronskian $W\left[u_{l}, w_{l}^{(+)}\right]$is unity. The $u_{l}$ is regular at the origin and $w_{l}^{(+)}$is an outgoing wave at infinity. The $l_{\max }=16$ is chosen in the later calculations.

We must choose the $V_{0}(\mathbf{r})$ so as to make the potential $\tilde{V}(\mathbf{r})=V(\mathbf{r})-V_{0}(\mathbf{r})$ be negligible outside the box. For neutral molecules, the attractive ionic potential is approximately canceled out by the repulsive direct Coulomb term. However we will later employ a gradient-corrected exchange potential which possesses a correct asymptotic form of $-e^{2} / r$. Therefore we use a jellium potential, Eq. 3.1), with $Z=1$ as $V_{0}(\mathbf{r})$. The $w_{l}^{(+)}(r ; E)$ in Eq. (2.30) is an irregular Coulomb wave function with an outgoing boundary condition. We use a FORTRAN77 program "COULCC" in Ref. 35 to calculate Coulomb functions of complex energies. The $u_{l}(r, E)$ in Eq. (2.31) is calculated by integrating the radial Schrödinger equation with a fourth-order Runge-Kutta method.

We now summarize our procedure to solve the response equation. Once an external field $V_{\text {ext }}(\mathbf{r}, \omega)$ is given, Equations (2.16) and (2.17) constitute a linear equation for the transition density $\delta n(\mathbf{r}, \omega)$. Discretizing on a uniform grid, we have a linear algebraic equation with dimensionality equal to the number of grid points. In order to solve the equation, we need to calculate actions of the $\chi_{0}$ on some functions, which is equivalent to calculate actions of the $G^{(+)} . \psi_{i}\left(\mathbf{r} ; E, V_{\mathrm{scf}}\right)$ is calculated by solving another linear equation (2.27). In order to solve Eq. 2.27), we need to calculate actions of the $G_{0}^{(+)}$. This operation is achieved by solving Eq. (2.29), again discretizing on grid points. We note that the outgoing boundary condition is imposed at this stage. The wave function $\Psi(\mathbf{r} ; E)$ outside the box is prepared by a multipole expansion method, Eqs. (2.30) and (2.31). Solutions of linear equations (2.16), (2.27) and (2.29) are obtained by iterative methods. The iterative procedure is summarized in Fig. 11.

Our algorithm thus requires to solve multiple nested linear algebraic equations. Conjugate gradient (CG) method and its variants offer stable and efficient scheme to solve these equations. If the energy $E$ is real, Eq.
(2.29) is a hermitian problem. Therefore, the CG method is very powerful to solve the equation. However, if the energy is complex, Eq. (2.29) becomes a non-hermitian problem to which the CG method is not applicable. For such cases, we adopt a Bi-conjugate gradient (Bi-CG) method. To solve Eq. (2.27), we have tested different iterative methods to this non-hermitian problem. We found that a generalized conjugate residual (GCR) method is one of the most effective algorithms for the present problem. For the outer most iteration loop of Eq. (2.23), we use the GCR method again.

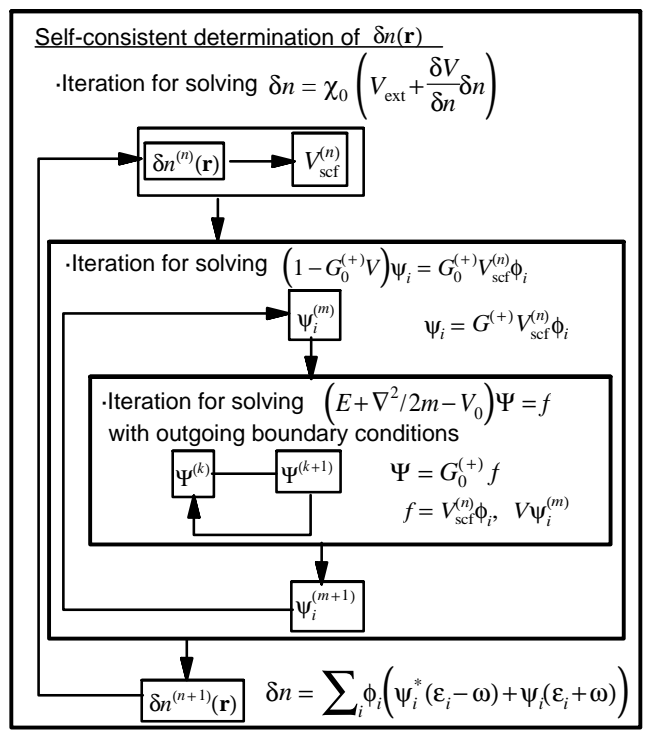

FIG. 1. Algorithm to determine the transition density $\delta n(\mathbf{r}, \omega)$ is presented. There are three nested iterative loops to solve linear equations.

\section{APPLICATIONS}

\section{A. Spherical jellium model for $\mathrm{Na}_{7}^{-}$: Test study}

First we have carried out a calculation for a negativelycharged $\mathrm{Na}$ cluster $\mathrm{Na}_{7}^{-}$. We use a spherical uniformlycharged jellium potential for $V_{\text {ion }}(r)$. The jellium potential of radius $R_{I}$ is given by

$$
V_{\text {ion }}(r)= \begin{cases}-\frac{Z e^{2}}{2 R_{I}}\left\{3-\left(\frac{r}{R_{I}}\right)^{2}\right\} & \text { for } r \leq R_{I}, \\ -\frac{Z e^{2}}{r} & \text { for } r>R_{I},\end{cases}
$$

where $Z e$ is a total charge of a jellium sphere. For the $\mathrm{Na}_{7}^{-}$, we have $Z=7$ and eight valence electrons. The main photoabsorption peak is calculated to be above the ionization threshold. Thus, this would be a good test case to study a response in the continuum and to check the applicability of the theories in the previous section.

We discretize three-dimensional Cartesian coordinates with spacing $\Delta x=\Delta y=\Delta z=1.5 \AA$ and employ grid 
points inside a spherical box of radius $R=12 \AA$. This is found enough for the ground-state density to be negligible at the edge $r=R$. The number of mesh points is 2109. The TDLDA Hamiltonian is given by Eq. (2.2) in which the exchange-correlation potential is that of Ref. 36]:

$$
\mu_{\mathrm{xc}}[n(\mathbf{r})]=-\frac{1.222}{r_{s}(\mathbf{r})}-0.0666 \ln \left(1+\frac{11.4}{r_{s}(\mathbf{r})}\right),
$$

in units of Ry where $4 / 3 \pi r_{s}^{3}=n(\mathbf{r})$. The radius of jellium sphere $R_{\mathrm{I}}$ is $3.93 \times 8^{1 / 3}$ a.u.

Since the jellium potential for $\mathrm{Na}_{7}^{-}$is spherical, we can use the same technique as that of Ref. [32,14] (a Fortran program in Ref. [16]) and confirm that our methods provide the same results. The Green's function is expanded in partial waves and is given by

$$
G_{l}^{(+)}\left(r, r^{\prime} ; E\right)=u_{l}\left(r_{<} ; E\right) w_{l}^{(+)}\left(r_{>} ; E\right) / W\left[u_{l}, w_{l}^{(+)}\right],
$$

for the $l$-th partial wave. Here $u_{l}$ and $w_{l}^{(+)}$are regular and irregular solutions of the radial differential equation, respectively. The boundary condition of $w_{l}^{(+)}$at the edge $r=R$ is defined as $\exp (i k r)$ with $k^{2}=2 m\left(E-e^{2} / R\right)$ for $E>e^{2} / R$ and $\exp (-\kappa r)$ with $\kappa^{2}=2 m\left(e^{2} / R-E\right)$ for $E<e^{2} / R$. Of course, this cause a change of the threshold energy by $e^{2} / R$, but it is enough to check validity of our methodology. We also add a small imaginary parameter $\Gamma / 2$ to the frequency $\omega$, which plays exactly the same role as a smoothing parameter of the Fourier transform in real-time method, Eq. (2.8). In this calculation, $\Gamma=0.1 \mathrm{eV}$ is used and the mesh size for radial coordinate is as small as $0.1 \AA$.

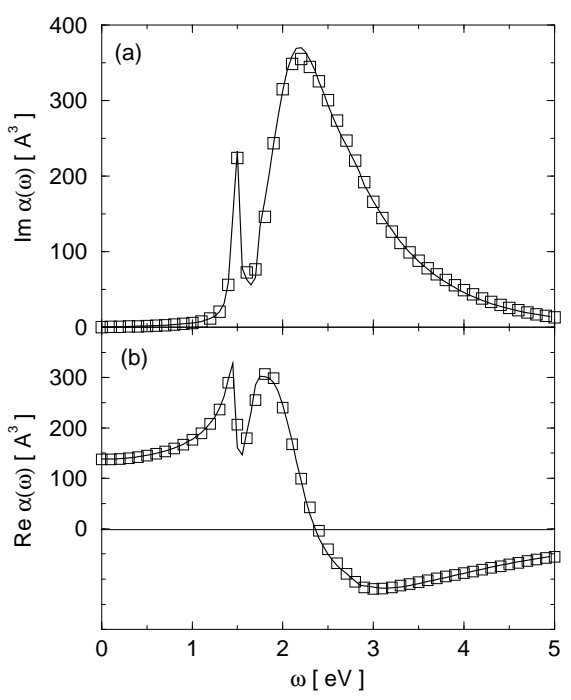

FIG. 2. Imaginary (a) and real (b) part of dynamic polarizabilities for $\mathrm{Na}_{7}^{-}$cluster calculated with the Green's function method. Solid lines are the results of one-dimensional calculation of Ref. [16 of jellium sphere and square symbols are those of the three-dimensional calculation without assuming any symmetry. The smoothing parameter $\Gamma=0.1 \mathrm{eV}$ is used for both calculations.
For the three-dimensional Green's function calculation, in order to justify Eq. (2.26), a condition that $\tilde{V}(\mathbf{r}) \approx 0$ for $r>R$ must be satisfied. We assume that the asymptotic behavior is the same as that of the (radial) onedimensional calculation. Namely, we adopt the Green's function of free particles, Eq. (2.25), with an energy being shifted as $E \rightarrow E-e^{2} / R$. We also use the same smoothing parameter $\Gamma=0.1 \mathrm{eV}$. It turns out that the inclusion of $\Gamma$ helps convergence of iterative procedure.

We show the results in Fig. 2. The three-dimensional calculation has been done for a frequency range $0<\omega<$ $5 \mathrm{eV}$ with a step $\Delta \omega=0.1 \mathrm{eV}$. The solid lines are the results of one-dimensional calculation in which the Green's functions are expanded in the partial wave, Eq. (3.3). The squares are the results of the three-dimensional calculation which perfectly agree with the solid lines. This means that the Green's function method on the threedimensional mesh is able to take account properly of the continuum effects.

The static calculation indicates the highest occupied molecular orbital (HOMO) $-0.37 \mathrm{eV}$, and a Coulomb potential has a value $e^{2} / R=1.2 \mathrm{eV}$ at the edge of the box $R=12 \AA$. Thus, the ionization threshold is $1.57 \mathrm{eV}$ in this calculation. The figure indicates that the photoabsorption has peaks below and above the threshold. In the IPA calculation, we obtain a single peak at an energy $1.4 \mathrm{eV}$. The screening potential brings the peak into the continuum region (around $2.35 \mathrm{eV}$ ) and splits the single peak into two parts at the threshold. The summed oscillator strengths for energies up to $5 \mathrm{eV}$ correspond to about $95 \%$ of the Thomas-Kuhn-Reiche (TRK) sum rule for valence electrons.

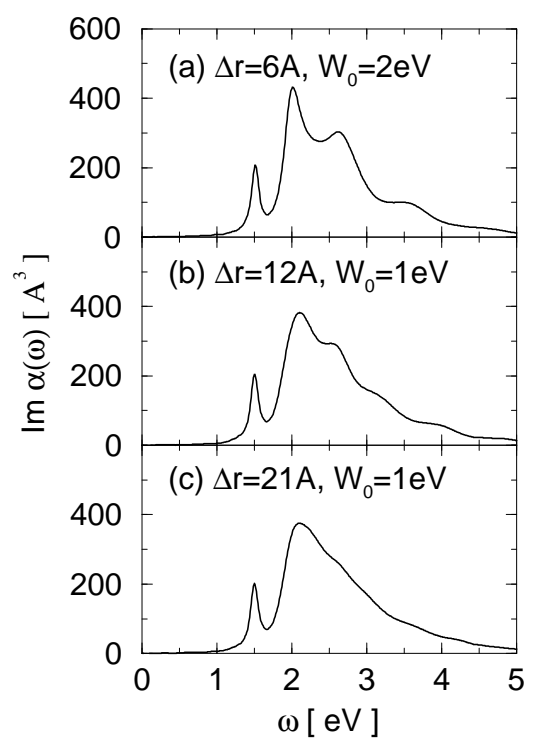

FIG. 3. Imaginary part of dynamic polarizabilities for $\mathrm{Na}_{7}^{-}$cluster calculated with the real-time method. The absorptive potentials are chosen as (a) $\Delta r=6 \AA$ with $W_{0}=2 \mathrm{eV}$, (b) $\Delta r=12 \AA$ with $W_{0}=1 \mathrm{eV}$, (c) $\Delta r=21$ $\AA$ with $W_{0}=1 \mathrm{eV}$. The smoothing parameter $\Gamma=0.1 \mathrm{eV}$ is used on the Fourier transform of Eq. (2.8). 
We now turn to the real-time method. A necessary size of space is much larger than that of the Green's function method because the absorbing potential is set up in a outer region $R<r<R+\Delta r$. We prepare three kinds of absorbing potentials in a linear form of Eq. (2.10): (a) $\Delta r=6 \AA$ with $W_{0}=2 \mathrm{eV}$, (b) $\Delta r=12 \AA$ with $W_{0}=1$ $\mathrm{eV}$ and (c) $\Delta r=21 \AA$ with $W_{0}=1 \mathrm{eV}$. Using a square mesh of $\Delta x=\Delta y=\Delta z=1.5 \AA$, the numbers of mesh points are (a) 7153, (b) 17077, and (c) 44473, respectively. We use a duration of time evolution $T=70 \mathrm{eV}^{-1}$ with a time step $\Delta t=0.01 \mathrm{eV}^{-1}$ and a smoothing parameter $\Gamma=0.1 \mathrm{eV}$ for the Fourier transform. Since we have a complex potential at the edge of the box, the energy is not strictly conserved but the scale of violation turns out to be less than $0.1 \%$ for this calculation. The imaginary part of dynamic polarizability is shown in Fig. 3 . Parts (a), (b) and (c) correspond to the three cases described above. For (a) and (b), the main resonance in the continuum region looks like a superposition of three different peaks which are located at $\omega \approx 2,2.7$ and 3.5 $\mathrm{eV}$, respectively. However, since we cannot see the same behavior in Fig. 2 (a), this should be a spurious effect of the reflection of outgoing waves. This is confirmed by a calculation of (c), for which the structure at $\omega>2.5 \mathrm{eV}$ is almost disappeared and we see only a smooth tail.

Now let us check the criterion of a good absorber, Eq. (2.15). The position of the main peak is calculated at $E_{\mathrm{res}}-E_{\mathrm{thr}}=2.35-1.57 \approx 0.8 \mathrm{eV}$ above the ionization threshold. Using $\mathrm{E}=0.8 \mathrm{eV}$ and $(8 m)^{-1} \approx 1 \mathrm{eV} \AA^{2}$ for electrons, Eq. (2.15) reduces to

$$
18(\Delta r)^{-1}<\left|W_{0}\right|<0.07 \Delta r
$$

where $W_{0}$ in units of $\mathrm{eV}$ and $\Delta r$ in $\AA$. This criterion is satisfied for the case (c) but not for (a) and (b). Fig. 3 shows that the real-time method provides us with a correct response in the continuum if no reflection occurs at the edge of the box. In order to find a suitable absorbing potential (of a linear form), we find the criterion, Eq. (2.15), very useful even when a condition $W_{0} / E \ll 1$ is not satisfied.

\section{B. Valence shell photoabsorption of silane}

It is well known that the energy of the highest occupied orbital does not coincide with the first ionization potential in the simplest local-density approximation. This fact causes a serious problem for the continuum response calculation that the ionization threshold cannot be adequately described by the static Kohn-Sham Hamiltonian. Furthermore, the excited states around the ionization threshold appear in too low excitation energies. To remedy this defect, a gradient correction for the exchangecorrelation potential has been proposed. We utilize the one constructed by van Leeuven and Baerends [37] which we denote as $\mu^{(\mathrm{LB})}$. It is so constructed that the potential has a correct $-e^{2} / r$ tail asymptotically. The energy of the highest occupied orbital also approximately coincides with the ionization potential. For small molecules, TDLDA calculations with this gradient correction have shown to give accurate description of discrete excitations in small molecules 38.

In the following sections III B, III C and IIID, we employ a sum of the exchange-correlation potentials of $\mu^{(\mathrm{PZ})}$ of Ref. 39] for the local-density part and $\mu^{(\mathrm{LB})}$ for the gradient correction. We should remark here that an accurate calculation of the gradient correction $\mu^{(\mathrm{LB})}(\mathbf{r})$ becomes difficult at far outside the molecule, because the $\mu^{(\mathrm{LB})}$ depends on $|\nabla n(\mathbf{r})| / n(\mathbf{r})^{4 / 3}$ which approaches to finite but both the numerator and the denominator approaches to zero at $r \rightarrow \infty$. Thus, we use an explicit asymptotic form, $\mu^{(\mathrm{LB})}(\mathbf{r})=-e^{2} / r$ for $r>R_{\mathrm{c}}$. In the followings, the $R_{\mathrm{c}}$ is chosen as $6.5 \AA$ for silane and $5.5 \AA$ for acetylene and ethylene.

When we evaluate the screening field in Eq. (2.17), we neglect the functional derivative of $\mu^{(\mathrm{LB})}(\mathbf{r})$ with respect to the density because this should be a small correction. For the real-time calculation, we make the same approximation. Namely the time-dependent exchangecorrelation potential is calculated by

$$
\begin{aligned}
\mu_{\mathrm{xc}}(\mathbf{r}, t) & \approx \mu_{\mathrm{xc}}\left[n_{0}(\mathbf{r})\right]+\left.\int d^{3} r^{\prime} \frac{\delta \mu_{\mathrm{xc}}(\mathbf{r})}{\delta n\left(\mathbf{r}^{\prime}\right)}\right|_{n=n_{0}} \delta n\left(\mathbf{r}^{\prime}, t\right) \\
& \approx \mu_{\mathrm{xc}}\left[n_{0}(\mathbf{r})\right]+\left.\int d^{3} r^{\prime} \frac{\delta \mu^{(\mathrm{PZ})}(\mathbf{r})}{\delta n\left(\mathbf{r}^{\prime}\right)}\right|_{n=n_{0}} \delta n\left(\mathbf{r}^{\prime}, t\right) .
\end{aligned}
$$

In this section, we discuss the application of our techniques to a deformed molecule, silane $\mathrm{SiH}_{4}$. We use the pseudopotentials which are calculated by the prescriptions of Refs. 25,26. For Si atom, we employ pseudopotentials for $s, p, d$ waves and take $d$-wave potential as a local part. For the hydrogen atom, we take $s$ and $p$ potentials with the latter as a local potential. The box is a sphere of radius $R=7 \AA$ discretized in meshes of $\Delta x=\Delta y=\Delta z=0.4 \AA$. Position of the Si atom (nucleus) is located at the origin and four hydrogen atoms are at $(1.209,0.0,0.855),(-1.209,0.0,0.855)$, $(0.0,1.209,-0.855)$, and $(0.0,-1.209,-0.855)$ in units of $\AA$. The symmetry of this molecule belongs to the tetrahedral $\left(T_{d}\right)$ point group. The valence electronic orbitals in the ground state are

$$
\left(3 a_{1}\right)^{2},\left(2 t_{2}\right)^{6}:{ }^{1} A_{1} \text {. }
$$

In actual calculations, we do not assume a priori the symmetry, but the degeneracies of electron orbitals according to Eq. (III B) naturally emerge after the minimization of the total energy. For this molecule, the dipole response does not depend on the direction of an external field. A smoothing parameter $\Gamma=0.5 \mathrm{eV}$ is used in the followings.

Utilizing the exchange-correlation potential of $\mu^{(\mathrm{PZ})}+$ $\mu^{(\mathrm{LB})}$, the calculated occupied valence orbitals in the ground state are listed in Table II. If we neglect the gradient correction term $\mu^{(\mathrm{LB})}$, we obtain $-13.5 \mathrm{eV}$ for $3 a_{1}$ 
and $-8.3 \mathrm{eV}$ for $2 t_{2}$, respectively. The photoabsorption oscillator strengths for silane calculated by means of the Green's function method are shown in Fig. 14. The oscillator strength $d f / d \omega$, the photoabsorption cross section $\sigma(\omega)$, and the imaginary part of dynamic polarizability $\operatorname{Im} \alpha(\omega)$, are related to each other by

$$
\sigma(\omega)=\frac{2 \pi^{2} e^{2}}{m c} \frac{d f}{d \omega}=\frac{4 \pi \omega}{c} \operatorname{Im} \alpha(\omega)
$$

A sharp peak at $10 \mathrm{eV}$ in the calculation consists of bound discrete peaks overlapped by the width $\Gamma$. In the experiment 43. 44. 1], we observe a peak at $10.7 \mathrm{eV}$, the width of which is considered to originate from a coupling of electronic excitation with ionic motion. Since we fix the ion coordinates and calculate vertical electronic excitations, we cannot describe the width below the ionization threshold.

On the other hand, a broad peak at $14.6 \mathrm{eV}$ in the experiment is above the ionization threshold and the main decay channel is an emission of the electron 443,1]. The calculation well reproduces this peak although the energy shifts slightly to the lower $(14 \mathrm{eV})$. Beyond the peak of $14.6 \mathrm{eV}$, a group of tiny peaks is observed in the experiment, which is assigned the Rydberg states with a hole $\left(3 a_{1}\right)^{-1}$ embedded in the continuum. This fine structure is smeared out in the calculation because the smoothing parameter $\Gamma$ is much larger than the width of each Rydberg state. The integrated oscillator strengths for energies up to $30 \mathrm{eV}$ is calculated to exhaust $87 \%$ of the TRK sum rule for valence electrons. About $60 \%$ of the valence shell strengths lie between 10 and $20 \mathrm{eV}$ (Fig. 5 (b)).

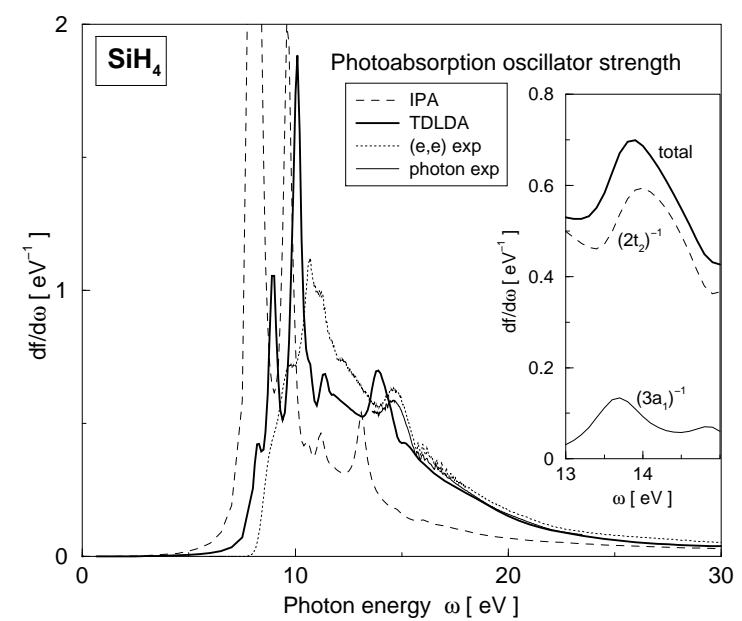

FIG. 4. Calculated and experimental photoabsorption oscillator strengths of silane. The thick solid line is the calculation compared with synchrotron radiation experiment (thin solid line) 43] and high resolution dipole (e,e) experiment (dotted line) 44. The dashed line is the IPA calculation without dynamical screening. The smoothing parameter $\Gamma=0.5 \mathrm{eV}$ is used in the calculation. Inset: An energy region of $13<\omega<15 \mathrm{eV}$ is magnified and the total oscillator strengths are decomposed into those associated with different occupied valence electrons, $3 a_{1}$ (solid line) and $2 t_{2}$ (dashed line).
The screening field in Eq. (2.17) significantly reduces a dipole polarizability at low frequency. The calculated static dipole polarizability is $5.1 \AA^{3}$ while it is $7.9 \AA^{3}$ in the IPA. The dielectric effects also change the absorption spectra. The screening shifts the resonance energies to higher energies because the electron-electron correlation is repulsive. Roughly speaking, the IPA overestimates the absorption spectra at low frequency and underestimates at high frequency. As you see in Fig. A, the dielectric effects are very important to reproduce the magnitude and shape of the photoresponse.

In order to discuss details of each resonance, it is useful to calculate a partial oscillator strength of each occupied orbital which should be identified with a photoemission spectra if the neutral dissociation channel without electron emission is negligibly small. As we see in Ref. 14], the photoabsorption cross section can be written as a sum of contributions from all occupied orbitals. Here we define the partial oscillator strengths $(d f / d \omega)_{i}$ as

$$
\begin{aligned}
& \frac{d f}{d \omega}=2 \sum_{i}^{\mathrm{occ}}\left(\frac{d f}{d \omega}\right)_{i}, \\
& \left(\frac{d f}{d \omega}\right)_{i}=-\frac{2 \omega}{\pi e^{2}} \int d^{3} r d^{3} r^{\prime} V_{\mathrm{scf}}^{*}(\mathbf{r}) \phi_{i}(\mathbf{r}) \times \\
& \operatorname{Im}\left\{\left(\tilde{G}^{(+)}\left(\mathbf{r}, \mathbf{r}^{\prime} ;\left(\epsilon_{i}-\omega\right)^{*}\right)\right)^{*}+\tilde{G}^{(+)}\left(\mathbf{r}, \mathbf{r}^{\prime} ; \epsilon_{i}+\omega\right)\right\} \\
& \times V_{\mathrm{scf}}\left(\mathbf{r}^{\prime}\right) \phi_{i}\left(\mathbf{r}^{\prime}\right),
\end{aligned}
$$

where $\tilde{G}^{(+)}$is defined by Eq. (2.19) except that the summation with respect to $k$ is carried out only for unoccupied states.

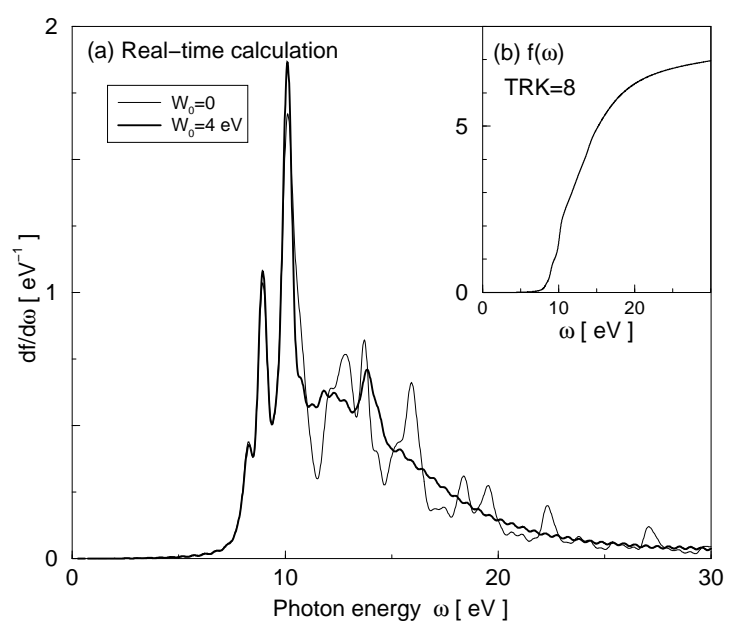

FIG. 5. (a) Photoabsorption oscillator strengths of silane calculated with the real-time method. The thin line corresponds to the result obtained with the box of $R=10$ $\AA$ without a complex absorbing potential, while the thick line to the one with a complex potential of $W_{0}=4 \mathrm{eV}$ with $\Delta r=10 \AA$ in addition to a spherical box of $R=7 \AA$. In carrying out the Fourier transform, the smoothing parameter $\Gamma=0.5 \mathrm{eV}$ is used. (b) The integrated oscillator strength $f(\omega)$. The TRK sum rule indicates $f(\infty)=8$ for the valence shell photoabsorption. 
TABLE I. Calculated eigenvalues of occupied valence orbitals and experimental vertical ionization potential (IP) in units of eV. The experimental data are taken from Ref. [40] for silane, from Ref. [41] for acetylene and from Ref. 42] for ethylene.

\begin{tabular}{|c|c|c|c|c|c|c|c|c|}
\hline \multicolumn{3}{|c|}{ Silane } & \multicolumn{3}{|c|}{ Acetylene } & \multicolumn{3}{|c|}{ Ethylene } \\
\hline $\begin{array}{c}\text { occ } \\
\text { state }\end{array}$ & cal & $\begin{array}{c}\text { exp } \\
\text { IP }\end{array}$ & $\begin{array}{c}\text { occ } \\
\text { state }\end{array}$ & cal & $\begin{array}{l}\text { exp } \\
\text { IP }\end{array}$ & $\begin{array}{c}\text { occ } \\
\text { state }\end{array}$ & cal & $\begin{array}{c}\text { exp } \\
\text { IP }\end{array}$ \\
\hline$\left(3 a_{1}\right)^{2}$ & -17.4 & 18.2 & $\left(2 \sigma_{g}\right)^{2}$ & $\overline{-22.4}$ & 23.5 & $\left(2 a_{q}\right)^{2}$ & -22.8 & $\overline{23.7}$ \\
\hline$\left(2 t_{2}\right)^{6}$ & -12.4 & 12.8 & $\left(2 \sigma_{u}\right)^{2}$ & -18.4 & 18.4 & $\left(2 b_{1 u}\right)^{2}$ & -18.6 & 19.4 \\
\hline & & & $\left(3 \sigma_{g}\right)^{2}$ & -16.7 & 16.4 & $\left(1 b_{2 u}\right)^{2}$ & -16.3 & 16.3 \\
\hline & & & $\left(1 \pi_{u}\right)^{4}$ & -12.1 & 11.4 & $\left(3 a_{g}\right)^{2}$ & -14.7 & 14.9 \\
\hline & & & & & & $\left(1 b_{3 g}\right)^{2}$ & -13.2 & 13.0 \\
\hline & & & & & & $\left(1 b_{3 u}\right)^{2}$ & -11.7 & 11.0 \\
\hline
\end{tabular}

Below the ionization threshold, there are three bound transitions in our calculations which we find are associated with the excitations of $2 t_{2}$ valence electrons. The first two transitions are considered to correspond to the shoulders in the measurements [44,45] and interpreted as transitions to the Rydberg states. The broad resonance at $14 \mathrm{eV}$ (14.6 eV in experiment) is above the ionization threshold of the $2 t_{2}$ orbitals. Therefore, the structure may be more complex. An inset of Fig. A shows the partial oscillator strengths of $3 a_{1}$ and $2 t_{2}$ orbitals in a photon-energy region of 13 to $15 \mathrm{eV}$. The calculation suggests that the resonance structure is due to the bound-to-bound excitation of $3 a_{1}$ electrons. The excitation couples with the bound-to-continuum excitations of $2 t_{2}$ electrons through the dynamical screening effect. The coupling shifts the excitation energy up by about 1 $\mathrm{eV}$ and brings about the width due to the autoionization process. The partial cross section of $2 t_{2}$ electrons also acquires oscillation as a function of the excitation energy due to the rapid change of the induced field.

Now let us examine the applicability of the real-time method. The time step is chosen as $\Delta t=0.002 \mathrm{eV}^{-1}$ and the time evolution is calculated up to $T=12 \mathrm{eV}^{-1}$. We show the results in Fig. 5 (a). The bound excited states is reasonably described in the calculation. On the other hand, the calculation without an absorbing potential (thin line) apparently provides a wrong response in the continuum region. We must adopt an imaginary potential to remove several spurious resonances. However, it is very difficult to treat the ionization energy properly. In order to mimic the continuum, the absorber should be effective only for a outgoing electron with positive energy. The problem is that the static potential has $-e^{2} / r$ behavior at large $r$. Electrons with negative energies $\left(-e^{2} / R<E<0\right)$ may be absorbed by the imaginary potential. Therefore, the effective ionization potential becomes $-\epsilon_{\text {HOMO }}-e^{2} / R$ for this calculation. Taking $R=7 \AA$, this shift in the ionization potential amounts to about $2 \mathrm{eV}$. The calculated peak in the continuum is located at $\omega=14 \mathrm{eV}$. According to the condition 2.15) with $E=14-12.4+2=3.6 \mathrm{eV}$, we adopt the $\Delta r=10 \mathrm{~A}$ and $W_{0}=4 \mathrm{eV}$. The number of grid points is 321,781 for this case. The thick line in Fig. 5 (a) shows the result. The spurious peaks disappear and the result well agrees with that of the Green's function method. The oscillator strengths near the ionization threshold $(11<\omega<13 \mathrm{eV})$ indicate some discrepancies, which we naturally expect from the above argument. In Fig. 5 (b), integrated oscillator strength is plotted against the energy. Seven out of eight (TRK sum rule) unit of strength locates below $30 \mathrm{eV}$.

Finally we should mention the feasibility of computation. In order to calculate the absorption spectra of Fig. 5, the real-time method takes about 10 hours using a single CPU of a Fujitsu VPP700E. On the other hand, the Green's function method takes about 30 minutes for each energy. Thus, the real-time method is faster than the Green's function method if the response is calculated for over 20 different energies. We have carried out the calculations for 125 frequencies to obtain the smooth line of Fig. 14.

\section{Valence shell photoabsorption of acetylene}

The acetylene molecule, $\mathrm{C}_{2} \mathrm{H}_{2}$, has a symmetry configuration of $D_{\infty h}$. This high symmetry has made possible a calculation of the Green's function using a single-center expansion [17]. Even so, only two kinds of molecular orbitals, $3 \sigma_{g}$ and $1 \pi_{u}$, which are primarily derived from atomic $p$ states have been taken into account in Ref. 17], because it was difficult to describe the $s$-derived states in the single-center formulation. In the present paper, we consider all valence orbitals, including the $2 \sigma_{g}$ and $2 \sigma_{u}$ in addition to the above $p$-derived orbitals, to calculate the photoresponses.

The spherical box is taken as $R=6 \AA$ with meshes of $\Delta x=\Delta y=\Delta z=0.3 \AA$. All atoms are located on the $z$-axis at $\pm 0.601 \AA$ for carbon and $\pm 1.663 \AA$ for hydrogen. There are ten valence electrons and the calculated energies of occupied orbitals are listed in Table I. Using the Green's function method, we obtain the photoabsorption oscillator strengths as a function of photon energy, shown in Fig. 6. The calculation indicates a sharp bound 
resonance at $\omega=9.6 \mathrm{eV}$ and a broad structure around $15 \mathrm{eV}$ which seems to be a superposition of three resonances. The resonance at $9.6 \mathrm{eV}$ strongly responds to a dipole field parallel to the molecular axis. The large oscillator strengths in the IPA at $\omega=5 \sim 8 \mathrm{eV}$ and at $\omega=12.5 \sim 13.5 \mathrm{eV}$ are shifted to higher energies by the dielectric effects. The agreements with the experimental data are significantly improved by the inclusion of the dynamical screening. The static dipole polarizability is also affected significantly: In the IPA calculation, the polarizabilities parallel $\left(\alpha_{\|}\right)$and perpendicular $\left(\alpha_{\perp}\right)$ to the molecular axis are $\alpha_{\|}=10.7 \AA^{3}$ and $\alpha_{\perp}=3.87$ $\AA^{3}$. The dynamic screening reduces these values to 4.79 $\AA^{3}$ and $2.77 \AA^{3}$, respectively, which well agree with the experimental values, $\alpha_{\|}=4.73$ and $\alpha_{\perp}=2.87 \AA^{3}$ 477.

We find some disagreements between the calculation and the experiment in Fig. 6. We observe two distinct peaks in the experiments for the broad resonance around $15 \mathrm{eV}$. However, the calculation indicates three peaks. The lowest $(13.2 \mathrm{eV})$ and the highest ones $(15.9 \mathrm{eV})$ are related with responses to a dipole field parallel to the molecular axis, while the middle one $(14.3 \mathrm{eV})$ is a response to the perpendicular field. We plot the partial oscillator strengths in an inset of Fig. 6. The lowest peak at $13.2 \mathrm{eV}$ consists of the transition of $3 \sigma_{g}$ valence orbitals. The middle peak at $14.3 \mathrm{eV}$ turns out to consist of contributions of $2 \sigma_{u}$ and $1 \pi_{u}$ orbitals. We need an energy shift of these strengths by about $1 \mathrm{eV}$ to reproduce the experiments. An accurate configuration interaction calculation with the Schwinger variational method is available for this molecule 48. This calculation succeeds to reproduce the double peak structure. The assignments are consistent to ours: $3 \sigma_{g}$ for lower and $2 \sigma_{u}$ for higher transitions.

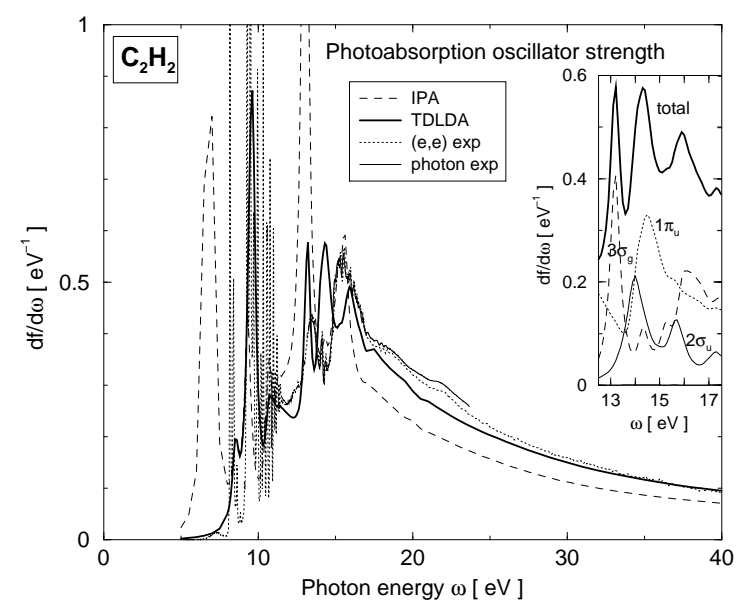

FIG. 6. Calculated and experimental photoabsorption oscillator strengths of acetylene. See the caption of Fig. 4. The experimental data are taken from Ref. 41,46. Inset: An energy region of $12.5<\omega<17.5 \mathrm{eV}$ is magnified and the total oscillator strength (thick line) is decomposed into partial oscillator strengths associated with different occupied valence orbital, $2 \sigma_{u}$ (solid line), $3 \sigma_{g}$ (dashed) and $1 \pi_{u}$ (dotted). The contributions of $2 \sigma_{g}$ electrons are negligible.

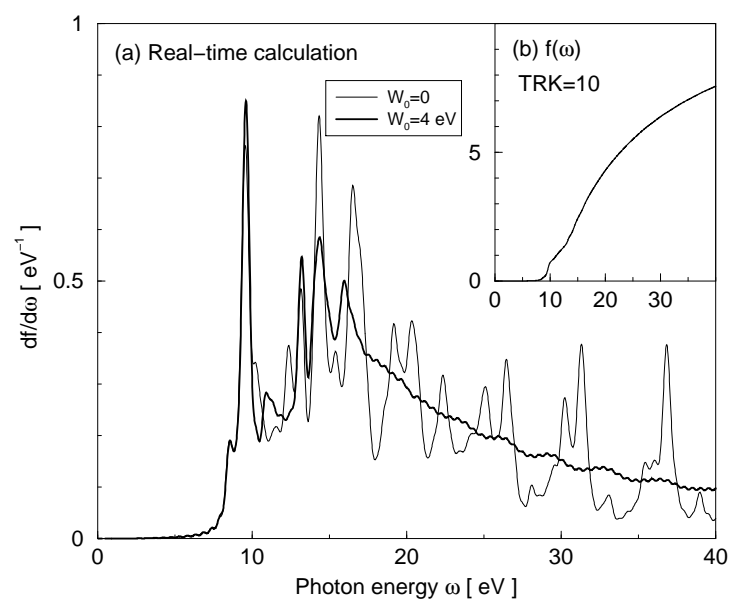

FIG. 7. (a) Photoabsorption oscillator strengths of acetylene calculated with the real-time method. The thin line corresponds to the result obtained with the box of $R=10 \AA$ without a complex absorbing potential, while the thick line to the one with a complex potential of $W_{0}=4 \mathrm{eV}$ and $\Delta r=10 \AA$ in addition to a spherical box of $R=6 \AA$. In carrying out the Fourier transform, the smoothing parameter $\Gamma=0.5 \mathrm{eV}$ is used. (b) The integrated oscillator strength $f(\omega)$. The TRK sum rule indicates $f(\infty)=10$ for the valence shell photoabsorption.

The experiments also indicate discrete Rydberg series around $10 \mathrm{eV}$. We do not see this structure in the calculation because we have used a smoothing parameter $\Gamma=0.5 \mathrm{eV}$ which is much larger than the experimental energy resolution. In principle, we can calculate these Rydberg states since the potential has a $-e^{2} / r$ behavior at large distance. In order to obtain these fine structures, we have to calculate the response with $\Gamma \approx 0$ and the small frequency mesh $\Delta \omega$ (order of meV) must be adopted. This is beyond a scope of this paper.

The real-time calculation is carried out with the same imaginary potential as we have used for silane $\left(W_{0}=4\right.$ $\mathrm{eV}$ and $\Delta r=10 \AA$ ). We have 635,371 grid points in this space. The time evolution is calculated up to $T=12$ $\mathrm{eV}^{-1}$ with a time step of $\Delta t=0.001 \mathrm{eV}^{-1}$. The results are shown in Fig. 7 (a). Again the absorbing potential is an essential ingredient to obtain sensible results in the continuum energy region. The calculated photoabsorption spectra well agree with those of the Green's function calculation except for minor oscillatory behaviors at high energy. This spurious oscillations start to appear at an energy $\omega \approx 22 \mathrm{eV}$. In fact, the condition of a good absorber, Eq. (2.15), is not satisfied in this energy region. Namely the potential $\left(W_{0}=4 \mathrm{eV}\right)$ is too week to absorb such high energy particles. In Fig. 7 (b), we show the integrated oscillator strengths up to $40 \mathrm{eV}$. One-fourth of the TRK sum rule value of valence electrons is in an energy region over $40 \mathrm{eV}$. It is worth noting that the CPU time of the real-time calculation is less than one-fifth of that of the Green's function calculation. 


\section{Valence shell photoabsorption of ethylene}

The ethylene $\mathrm{C}_{2} \mathrm{H}_{4}$ is the simplest organic $\pi$-system, which possesses the $D_{2 h}$ symmetry. The two carbon atoms are on the $x$-axis at $x= \pm 0.6695 \AA$ and four hydrogen atoms are in the $x-y$ plane at $(x, y)=$ $(1.2342,0.9288), \quad(1.2342,-0.9288), \quad(-1.2342,0.9288)$, $(-1.2342,-0.9288)$ in units of $\AA$. There are twelve valence electrons, and calculated eigenenergies of occupied valence orbitals are listed in Table $[$.

The calculations are carried out using the same box $(R=6 \AA)$ and mesh size $(0.3 \AA)$ as we have used for the acetylene molecule. The photoabsorption oscillator strengths calculated with the Green's function method are shown in Fig. 8. The agreement with an experiment 49 is excellent. Almost all the main features of photoabsorption spectra are reproduced in the calculation. The observed bound excited states show different photoresponses according to the direction of the dipole field. The lowest peak at $\omega=7.6 \mathrm{eV}$ is mainly a response to a dipole field parallel to the molecular $(\mathrm{C}-\mathrm{C})$ axis. This is associated with the excitations of the HOMO $1 b_{3 u}$ electrons. On the other hand, states at $9.8 \mathrm{eV}$ respond almost equally to a dipole field of $x, y$, and $z$ direction, to which both $1 b_{3 g}$ and $1 b_{3 u}$ occupied orbitals contribute. A small peak at $\omega=11.4 \mathrm{eV}$ is calculated as a resonance of $3 a_{g}$ orbital, which may correspond to a small shoulder in the experiment. Beyond $11.7 \mathrm{eV}$, the HOMO electrons are in the continuum. The first prominent peak at $12.4 \mathrm{eV}$ is a resonance with respect to a dipole field of $y$ direction which is in the molecular plane and perpendicular to the $\mathrm{C}-\mathrm{C}$ bond. The excitations of the $1 b_{2 u}$ and $1 b_{3 g}$ electrons are main components of this resonance. In a region of $13.2<\omega<20 \mathrm{eV}$, the $1 b_{3 g}$ electrons can be excited into the continuum and produce the smooth background of oscillator strengths $\left(0.1 \sim 0.2 \mathrm{eV}^{-1}\right)$. A peak structure at $14.6 \mathrm{eV}$ is made of the excitation of $1 b_{2 u}$ electrons. The peak at $16.4 \mathrm{eV}$ is constructed by excitations from the $2 b_{1 u}$ occupied orbitals, while the experiment indicates the resonance at $17.1 \mathrm{eV}$.

The above analysis is consistent with that in the literature [49], except for the $12.4 \mathrm{eV}$ peak $(11.9 \mathrm{eV}$ in the experiment). Our analysis suggests transition of $1 b_{3 g}$ and $1 b_{2 u}$ electrons while Ref. 449 indicates $3 a_{g}$.

Comparing the TDLDA calculation with that of the IPA (dashed line), we see again that the dynamic screening effect is very important to reproduce the experimental data. This feature of the IPA result is consistent with the other calculations without the dynamic screening [50]. The calculated dipole polarizability is $\alpha=4.22 \AA^{3}(5.47$, $3.97,3.23 \AA^{3}$ for $x, y, z$ direction, respectively), which is in a good agreement with the experimental value, 4.22 $\AA^{3}$ [47]. In the IPA calculation, we obtain $\alpha=7.04 \AA^{3}$ $(10.4,5.96,4.76)$.

Results of the real-time calculation are shown in Fig. 9 (a). The box and imaginary potential we use are the same as those for acetylene. We can obtain a sensible re- sult if we adopt the absorbing potential. However, again, a spurious oscillatory behavior is seen at high energy region because the absorbing potential is not strong enough to erase those high-energy components.

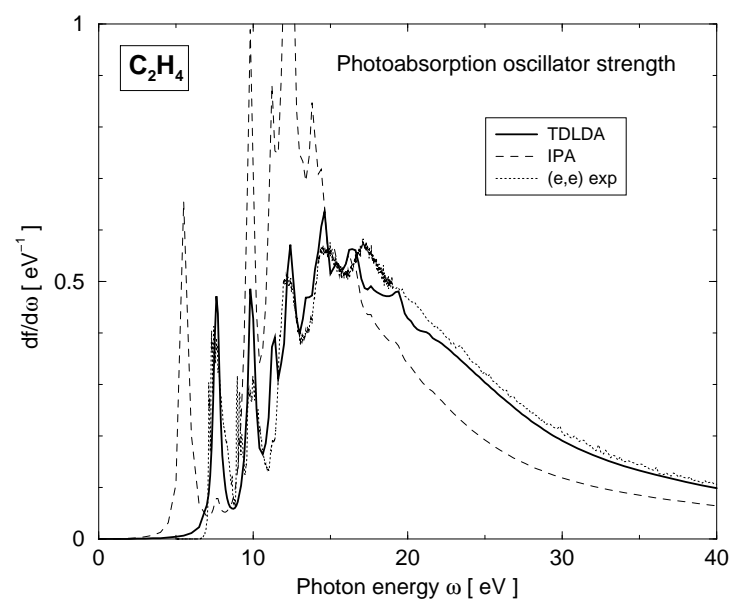

FIG. 8. Calculated and experimental photoabsorption oscillator strengths of ethylene. The thick solid line is the calculation compared with high resolution dipole (e,e) experiment (dotted line) [49]. The dashed line is the IPA calculation without dynamical screening. The smoothing parameter $\Gamma=0.5 \mathrm{eV}$ is used in the calculation.

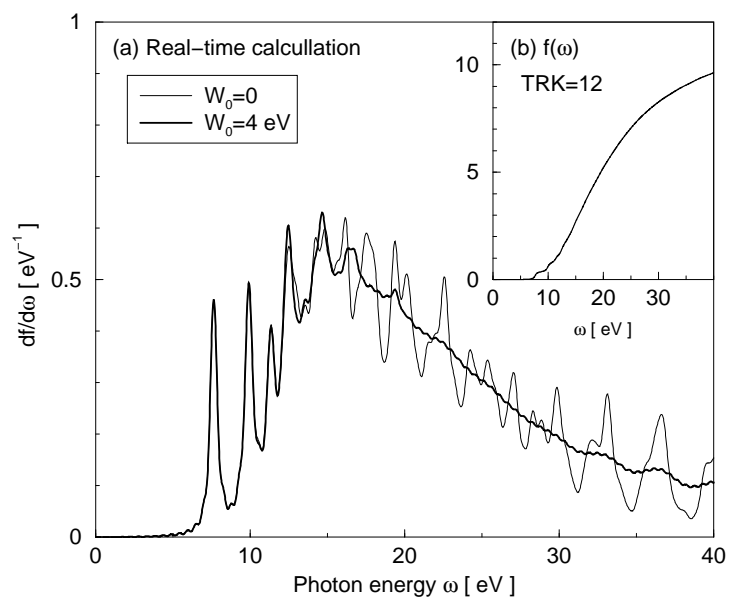

FIG. 9. Photoabsorption oscillator strengths of ethylene calculated with the real-time method. See the caption of Fig. 月 (a). (b) The integrated oscillator strength $f(\omega)$. The TRK sum rule indicates $f(\infty)=12$ for the valence shell photoabsorption.

\section{CONCLUSIONS}

We have developed methods based on the TDLDA of investigating the photoresponses in the continuum for systems with no spatial symmetry: (1) The real-time method with an absorbing potential, and (2) the Green's function method. These methods allow us to treat the photoionization and the dynamical screening effects selfconsistently. For the real-time method, we have tested 
imaginary potentials of different kinds and found that those satisfying a condition, Eq. (2.15), are good to mimic the continuum effect. However, it is very difficult to treat the photoabsorption spectra in vicinity of the ionization threshold. There are two reasons: One is because the condition, Eq. (2.15), requires a large model space to treat such a low-energy emission properly. The second is that the ionization threshold is not correct when the potential has a $1 / r$ behavior at large distance. In addition to this, since the condition is energy dependent, it is very difficult to construct a good absorber for low-energy and high-energy outgoing electrons simultaneously. The advantage of the real-time method is a computational feasibility. Utilizing the Fourier transform, we can obtain the spectra over the whole energy region at once.

The Green's function method possesses a capability of an exact treatment of the continuum. Using a Green's function of Coulomb asymptotic waves, it is also possible to investigate the photoresponse near the threshold. The main difficulty of the Green's function treatment is a heavy computational task. In order to reduce the CPU time, we use a complex energy for the Green's function, $G(\omega+i \Gamma / 2)$. We have found that the inclusion of the imaginary part $\Gamma$ facilitates a convergence of iterative procedures in the calculation. The $\Gamma$ also plays a role of lowering an energy resolution of the calculations. Thus, we can choose a value of $\Gamma$ depending on the energy resolution required in each problem. We would like to emphasize again that the method is capable of calculating response functions of many-electron systems, below, near and above the ionization threshold in a unified manner.

The numerical calculations have been performed for a spherical $\mathrm{Na}_{7}^{-}$cluster (test study) and non-spherical molecules, $\mathrm{SiH}_{4}, \mathrm{C}_{2} \mathrm{H}_{2}$, and $\mathrm{C}_{2} \mathrm{H}_{4}$. Studies of photoresponse for deformed molecules including both the dynamic screening and the continuum effect are very few. An exception is a study of nitrogen and acetylene molecules (with a high symmetry $D_{\infty h}$ ) by Levine and Soven 17] using the single-center expansion technique. However, only $1 \pi_{u}$ and $3 \sigma_{g}$ occupied orbitals are included in their calculation because of limitation of the singlecenter expansion. Since our calculation has been carried out on three-dimensional coordinate meshes, we do not need any spatial symmetry including all valence orbitals in the calculation. We present the photoabsorption oscillator strengths compared with dipole $(e, e)$ and synchrotron radiation experiments. The agreement is generally excellent. The inclusion of the dynamic screening turns out to be essential to reproduce the experiments. The IPA calculation overestimates the strengths at low energy and underestimates at high energy.

We are strongly encouraged by the success of our methods applied to simple molecules in the present paper. The applications to more complex systems may become possible in near future.

\section{ACKNOWLEDGMENTS}

We would like to thank Y. Hatano and K. Kameta for useful discussion and providing us with the photoabsorption experimental data. Calculations were performed on a Fujitsu VPP700E Super Computer at RIKEN, a NEC SX-4 Super Computer at Osaka University and a HITACHI SR8000 at Institute of Solid State Physics, University of Tokyo.

[1] Y. Hatano, Phys. Rep. 313, 109 (1999).

[2] I. Cacelli, V. Carravetta, A. Rizzo, R. Moccia, Phys. Rep. 205, 283 (1991).

[3] D. Dill and J. L. Dehmer, J. Chem. Phys. 61, 692 (1974).

[4] R. R. Lucchese, G. Raseev, and V. McKoy, Phys. Rev. A25, 2572 (1982).

[5] B. Basden and R. R. Lucchese, Phys. Rev. A37, 89 (1988).

[6] H. Le Rouzo and G. Raseev, Phys. Rev. A29, 1214 (1984).

[7] L. A. Collins and B. I. Schnieder, Phys. Rev. A29, 1695 (1984).

[8] I. Cacelli, V. Carravetta, R. Moccia, J. Chem. Phys. 85, 7038 (1986)

[9] P. W. Langoff, in Theory and Application of Moment Methods in Many-Fermion Systems, eds B.J. Dalton, S.M. Grimes, J.P. Vary and S.A. Williams (Plenum, New York, 1980), p191.

[10] V. Carravetta, L. Yang, and H. Ågren, Phys. Rev. B55, 10044 (1997).

[11] J. L. Pascual, L. G. M. Pettersson, and H. Ågren, Phys. Rev. B56, 7716 (1997).

[12] C. Yu, R. M. Pitzer, and C. W. McCurdy, Phys. Rev. A32, 2134 (1985).

[13] E. Runge and E. K. U. Gross, Phys. Rev. Lett. 52, 997 (1984).

[14] A. Zangwill and P. Soven, Phys. Rev. A 21, 1561 (1980).

[15] W. Ekardt, Phys. Rev. Lett. 52, 1925 (1984).

[16] G. Bertsch, Comp. Phys. Comm. 60, 247 (1990).

[17] Z. H. Levine and P. Soven, Phys. Rev. A 29, 625 (1984).

[18] J. R. Chelikowsky, N. Troullier, K. Wu, and Y. Saad, Phys. Rev. B50, 11355 (1994).

[19] K. Yabana and G. F. Bertsch, Phys. Rev. B54, 4484 (1996).

[20] K. Yabana and G. F. Bertsch, Int. J. Quantum Chem. 75, 55 (1999).

[21] G. D. Mahan, Phys. Rev. A22, 1780 (1980).

[22] Z. H. Levine, D. C. Allan, Phys. Rev. B43, 4187 (1991).

[23] J.-I. Iwata, K. Yabana, and G. F. Bertsch, Nonlinear Optics, 26, 9 (2000).

[24] F. Calvayrac, P.-G. Reinhard, E. Suraud, and C. A. Ullrich, Phys. Rep., in press.

[25] N. Troullier and J. L. Martins, Phys. Rev. B 43, 1993 (1991). 
[26] L. Kreinman and D. Bylander, Phys. Rev. Lett. 48, 1425 (1982).

[27] H. Flocard, S. Koonin, and M. Weiss, Phys. Rev. C 17, 1682 (1978).

[28] J. W. Negele, Rev. Mod. Phys. 54, 913 (1982).

[29] D. Neuhasuer and M. Baer, J. Chem. Phys. 90, 4351 (1989).

[30] M. S. Child, Mol. Phys. 72, 89 (1991).

[31] G. Bertsch and S. F. Tsai, Phys. Rep. 18, 125 (1975).

[32] S. Shlomo and G. Bertsch, Nucl. Phys. A243, 507 (1975).

[33] M. J. Stott and E. Zaremba, Phys. Rev. A 21, 12 (1980).

[34] A. L. Fetter and J. D. Walecka, Quantum Theory of Many-Body Systems (McGraw-Hill, New York 1971), Sec. 13.

[35] I. J. Thompson and A. R. Barnett, Comp. Phys. Comm. 36, 363 (1985).

[36] O. Gunnarsson and B. I. Lundqvist, Phys. Rev. B 13, 4274 (1976).

[37] R. van Leeuwen and E. J. Baerends, Phys. Rev. A 49, 2421 (1994).

[38] M. E. Casida, C. Jamorski, K. C. Casida, and
D. R. Salahub, J. Chem. Phys. 108, 4439 (1998).

[39] J. Perdew and A. Zunger, Phys. Rev. B 23, 5048 (1981).

[40] A. W. Potts and W. C. Price, Proc. R. Soc. London A 326, 165 (1972).

[41] M. Ukai et al., J. Chem. Phys. 95, 4142 (1991).

[42] D. M. P. Holland et al., Chem. Phys. 219, 91 (1997).

[43] K. Kameta et al., J. Chem. Phys. 95, 1456 (1991).

[44] G. Cooper, G. R. Burton, W. Fat Chan, and C. E. Brion, Chem. Phys. 196, 293 (1995)

[45] C. Y. R. Wu, F. Z. Chen, and D. L. Judge, J. Chem. Phys. 99, 1530 (1993).

[46] G. Cooper, G. R. Burton, and C. E. Brion, J. Electron Spectroy. Relat. Phenom. 73, 139 (1995).

[47] N. J. Bridge and A. D. Buchkingham, Proc. R. Soc. A 295, 334 (1966).

[48] M. C. Wells, and R. R. Lucchese, J. Chem. Phys. 111 (1999) 6290.

[49] G. Cooper, T. N. Olney, and C. E. Brion, J. Chem. Phys. 194, 175 (1995).

[50] F. A. Grimm, Chem. Phys. 81, 315 (1983). 